\title{
Magnetoelectric effect in a hydrogen molecule
}

\author{
Sylvain D. Brechet*, Francois A. Reuse, Klaus Maschke and Jean-Philippe Ansermet \\ Institute of Condensed Matter Physics, Station 3, EPFL, \\ 1015 Lausanne, Switzerland \\ *sylvain.brechet@epfl.ch
}

Received 7 January 2014

Revised 13 March 2014

Accepted 14 March 2014

Published 7 April 2014

\begin{abstract}
The symmetry breaking due to a magnetic field applied on a hydrogen molecule $\mathrm{H}_{2}$ generates an electric polarization. This magnetoelectric effect occurs for electrons in a triplet state provided the magnetic induction field is not aligned with the symmetry axis of the molecule.
\end{abstract}

Keywords: Magnetoelectric effect; hydrogen molecule; symmetry breaking.

\section{Introduction}

In the last few years, there was a revival for magnetoelectric effects ${ }^{1}$ especially in multiferroics ${ }^{2}$ or organics. ${ }^{3}$ Recently, magnetoelectric effects were reported to yield even realistic switching mechanisms in multiferroics. ${ }^{4}$

On the other hand, the energy levels of a hydrogen molecule in the presence of an external magnetic induction field were first examined by Ramsey ${ }^{5}$ and for superstrong magnetic fields, they were subsequently investigated by Lai et al. ${ }^{6}$ The ground state as a function of an external magnetic induction field was determined by Detmar et al. ${ }^{7}$ and for superstrong magnetic fields by Ortiz et al. ${ }^{8}$ The proof of stability of the hydrogen molecule was established by Richard et al. ${ }^{9}$ and the stability of molecular hydrogen in strong magnetic fields was investigated subsequently by Beau et al. ${ }^{10}$ The effect of the change of orientation of the external magnetic induction field on the hydrogen molecule was studied by Basile et al. ${ }^{11}$ using linear combination of atomic orbital (LCAO) methods.

Here, we present what we believe is the simplest possible molecular model for magnetoelectric effect. This model is based on the spatial axisymmetry breaking of a hydrogen molecule due to the presence of an external magnetic induction field.

A hydrogen molecule consists of two atomic sites containing a proton each and two electrons in a bound state around the atomic sites. In the absence of an 


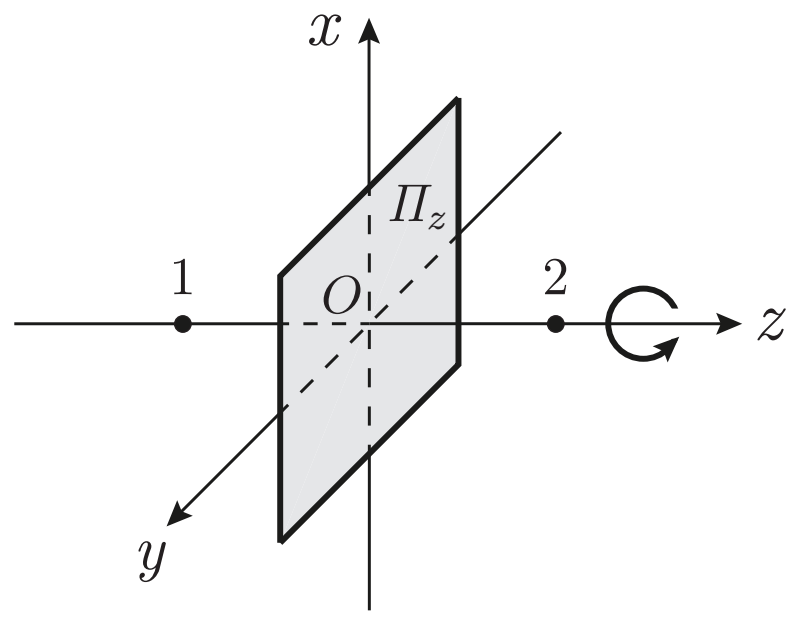

Fig. 1. Symmetries of the $H_{2}$ molecule with two atomic sites $(1,2)$

external interaction, the hydrogen molecule has a reflection symmetry and a rotation symmetry along the axis joining the sites. The hydrogen molecule $\mathrm{H}_{2}$ is oriented such that it is axisymmetric with respect to the $z$ axis and symmetric with respect to the $\Pi_{z}$ plan, that is orthogonal to the $z$ axis and contains the origin $O$. The two atomic sites of the hydrogen molecule $H_{2}$ denoted 1 and 2 are located on the $z$ axis at equal distance of the origin $O$, as shown in Fig. 1.

\section{Magnetoelectric Effect Generated by Symmetry Breaking}

In this paper, we make use of the Born-Oppenheimer approximation. ${ }^{12}$ In that approximation, the sites are motionless and the distance between the sites is assumed to be fixed. Moreover, we only consider the case of electrons that are in a triplet state. In such a state, the total spin of the electrons vanish, i.e. $\hat{\mathbf{S}}=\mathbf{0}$. Thus, we need to consider only the spatial part of the state. The reflection operator $\hat{\Pi}_{z}$ is a unitary operator that satisfies the following property,

$$
\hat{\Pi}_{z}=\hat{\Pi}_{z}^{\dagger}=\hat{\Pi}_{z}^{-1} .
$$

Under the action of the reflection operator $\hat{\Pi}_{z}$, the $z$ component of a vectorial operator changes sign, but the $x$ and $y$ components remain unchanged, and vice versa for a pseudo-vectorial operator. Thus, under the action of $\hat{\Pi}_{z}$, the components of the position operator $\hat{\mathbf{r}}$, of the momentum operator $\hat{\mathbf{p}}$ and of the angular momentum operator $\hat{\mathbf{L}}$ transform respectively as, ${ }^{13}$

$$
\begin{array}{lll}
\hat{\Pi}_{z} \hat{r}_{x, y} \hat{\Pi}_{z}=\hat{r}_{x, y} & \text { and } & \hat{\Pi}_{z} \hat{r}_{z} \hat{\Pi}_{z}=-\hat{r}_{z}, \\
\hat{\Pi}_{z} \hat{p}_{x, y} \hat{\Pi}_{z}=\hat{p}_{x, y} & \text { and } & \hat{\Pi}_{z} \hat{p}_{z} \hat{\Pi}_{z}=-\hat{p}_{z}, \\
\hat{\Pi}_{z} \hat{L}_{x, y} \hat{\Pi}_{z}=-\hat{L}_{x, y} & \text { and } & \hat{\Pi}_{z} \hat{L}_{z} \hat{\Pi}_{z}=\hat{L}_{z}
\end{array}
$$


In order satisfy the symmetries of the hydrogen molecule, the free Hamiltonian $\hat{H}_{0}$ commutes with the reflection operator $\hat{\Pi}_{z}$, i.e.

$$
\left[\hat{H}_{0}, \hat{\Pi}_{z}\right]=\hat{0} .
$$

In the presence of a constant external magnetic induction field $\mathbf{B}$, the interaction between the magnetic induction field $\mathbf{B}$ and the orbital angular momentum $\hat{\mathbf{L}}$ of each electron leads to a Zeeman effect $^{14}$ that is described by the interaction Hamiltonian,

$$
\hat{H}_{\mathrm{int}}=-\left(\frac{e}{2 m_{e}} \mathbf{B} \cdot \hat{\mathbf{L}}\right) \otimes \mathbb{1}-\mathbb{1} \otimes\left(\frac{e}{2 m_{e}} \mathbf{B} \cdot \hat{\mathbf{L}}\right),
$$

where $m_{e}$ is the mass of an electron, $e$ is the electric charge of a proton and the tensorial product relates the single electron subsystems.

The commutation relation between the interaction Hamiltonian $\hat{H}_{\text {int }}$ and the reflection operator $\hat{\Pi}_{z}$ yields,

$$
\left[\hat{H}_{\mathrm{int}}, \hat{\Pi}_{z}\right]=-\frac{e}{m_{e}}\left(B_{x} \hat{L}_{x}+B_{y} \hat{L}_{y}\right) \otimes \mathbb{1}-\mathbb{1} \otimes \frac{e}{m_{e}}\left(B_{x} \hat{L}_{x}+B_{y} \hat{L}_{y}\right) .
$$

Thus, the interaction Hamiltonian $\hat{H}_{\text {int }}$ commutes with the reflection operator $\hat{\Pi}_{z}$ only if the external magnetic induction field $\mathbf{B}$ is directed along the $z$ axis. Otherwise, the reflection symmetry is broken. If the reflection symmetry is broken, there is a resulting electric polarization $\mathbf{P}$ of the hydrogen molecule. Thus, a constant external magnetic induction field $\mathbf{B}$ that has a non-vanishing component along the $x$ or $y$ axis generates an electric polarization $\mathbf{P}$ of the hydrogen molecule, which is a magnetoelectric effect.

\section{Conclusion}

Finally, we showed that an electric polarization $\mathbf{P}$ can be generated on an hydrogen molecule by applying an external magnetic induction field $\mathbf{B}$ that is not collinear to the symmetry axis of the molecule. This is a simple yet elegant magnetoelectric effect that relies exclusively on symmetry breaking. The numerical estimation of the electric polarization of the $\mathrm{H}_{2}$ would require an explicit computation using the LCAO method, ${ }^{15}$ which extends far beyond the present work.

\section{References}

1. M. Fiebig, J. Phys. D: Appl. Phys. 38(8) (2005) 123.

2. C.-W. Nan, M. I. Bichurin, S. Dong, D. Viehland and G. Srinivasan, J. Appl. Phys. 103(3) (2008) 031101.

3. W. Wang et al., Sci. Rep. 3 (2013) 2024.

4. Y. Yang, J. Íñiguez, A.-J. Mao and L. Bellaiche, Phys. Rev. Lett. 112 (2014) 057202.

5. N. F. Ramsey, Phys. Rev. 85 (1952) 60.

6. D. Lai and E. E. Salpeter, Phys. Rev. A 53 (1996) 152.

7. T. Detmer, P. Schmelcher, F. K. Diakonos and L. S. Cederbaum, Phys. Rev. A 56 (1997) 1825. 
S. D. Brechet et al.

8. G. Ortiz, M. D. Jones and D. M. Ceperley, Phys. Rev. A 52 (1995) 3405.

9. J.-M. Richard, J. Fröhlich, G.-M. Graf and M. Seifert, Phys. Rev. Lett. 71 (1993) 1332 .

10. M. Beau, R. Benguria, R. Brummelhuis and P. Duclos, J. Phys. A: Math. Theor. 43(47) (2010) 474005.

11. S. Basile, F. Trombetta and G. Ferrante, Il Nuovo Cimento D 9(5) (1987) 457.

12. M. Born and R. Oppenheimer, Ann. Phys. 389 (1927) 457.

13. P. Jacobs, Group Theory with Applications in Chemical Physics (Cambridge University Press, 2005).

14. L. Ballentine, Quantum Mechanics: A Modern Development (World Scientific Publishing, 1998).

15. J. E. Lennard-Jones, Trans. Faraday Soc. 25 (1929) 668. 